\title{
Immortalized Cancer-associated Fibroblasts Promote Prostate Cancer Carcinogenesis, Proliferation and Invasion
}

\author{
SHENGQIANG YU ${ }^{1 *}$, YINGJUAN JIANG $^{2 *}$, FENGCHUN WAN $^{1 *}$, \\ JITAO WU ${ }^{1}$, ZHENLI GAO $^{1}$ and DONGFU LIU ${ }^{1}$ \\ ${ }^{1}$ Department of Urology, the Affiliated Yantai Yuhuangding Hospital of Qingdao University, Yantai, P.R. China; \\ ${ }^{2}$ Yantai Maternal and Child Health Hospital, Yantai, P.R. China
}

\begin{abstract}
Background: Cancer-associated fibroblasts (CAFs) are dominant components of the prostate cancer (PCa) stroma. However, the contrasting effects of CAFs and adjacent normal prostate fibroblasts (NPFs) are still poorly defined. The senescence of non-immortalized CAFs after subculture may limit the cell number and influence experimental results of in vitro studies. In this study, we immortalized CAFs to study their role in PCa carcinogenesis, proliferation, and invasion. Materials and Methods: We cultured and immortalized CAFs and NPFs, then compared their effect on epithelial malignant transformation by using in vitro co-culture, soft agar assay, and a mouse renal capsule xenograft model. We also compared their roles in PCa progression by using in vitro co-culture, cell viability assays, invasion assays, and a mouse xenograft model. For the mechanistic study, we screened a series of growth factors by using real-time polymerase chain reaction. Results: The CAFs and NPFs were successfully cultured, immortalized, and characterized. The CAFs were able to transform prostate epithelial cells into malignant cells, but NPFs were not. The $C A F s$ were more active in promoting proliferation of and invasion by PCa cells, and in secreting higher levels of a series of growth factors. Conclusion: The immortalized CAFs were more supportive of PCa carcinogenesis and progression. Targeting CAFs might be a potential option for PCa therapy. Immortalized CAFs and NPFs will also be valuable resources for future experimental exploration.
\end{abstract}

\footnotetext{
*These Authors contributed equally to this study.

Correspondence to: Dr. Dongfu Liu, Department of Urology, the Affiliated Yantai Yuhuangding Hospital of Qingdao University, 20 Yuhuangding East Road, Yantai, Shandong 264000, China. Tel/Fax: +86 5356695579, e-mail: 18561116833@163.com
}

Key Words: Immortalized cancer-associated fibroblasts, carcinogenesis, proliferation and invasion.
Prostate cancer (PCa) is one of the most common cancers in developed countries, and its incidence is increasing in China $(1,2)$. Radical prostatectomy and radiotherapy are generally recommended for localized $\mathrm{PCa}$, whilst a comprehensive therapy based on androgen deprivation therapy is first-line treatment for advanced disease (2). Although initially effective at blocking tumor growth, androgen deprivation therapy can eventually fail, leading to a castration-resistant incurable disease stage (3). The current therapeutic options for advanced PCa mostly target cancer cells. However, prostate stromal cells also play key roles in PCa progression (4).

Prostate cancer-associated fibroblasts (CAFs) are dominant components in PCa stroma, and have been reported to promote proliferation of epithelial cells $(5,6)$. In a previous study, we successfully cultured CAFs from $\mathrm{PCa}$ tissues and found that androgen receptors in CAFs promoted PCa epithelial growth and invasion (7). However, the contrasting effects of CAFs and adjacent normal prostate fibroblasts (NPFs) remain poorly defined.

Most researchers use primary CAFs without immortalization. Non-immortalized CAFs can only be subcultured for 6-8 passages, and different sets of primary cells from different $\mathrm{PCa}$ tissues may influence the consistency of experimental results. In this study, we primarily cultured and immortalized CAFs and NPFs from the same patient with PCa. Subsequently, we compared their effects on malignant transformation and progression of $\mathrm{PCa}$ by using in vitro experiments and in vivo mouse xenograft models. We also compared expression patterns of growth factors between CAFs and NPFs.

\section{Materials and Methods}

Cell culture. PCa specimens and normal prostate tissues were obtained from a patient with PCa (67 years old, primary PCa, Gleason score $=3+3$ ) at the Urology Department of Yantai Yuhuangding Hospital. The sample collection and primary cell culture were approved by the Yantai Yuhuangding Hospital Ethics Committee (Approval No. 2013-46). The patient was well informed and agreed to the use of his samples for cell culture and further 
research. All procedures abide by the 1964 Helsinki declaration and its later amendments or comparable ethical standards. The detailed procedure of the primary culture of CAFs and NPFs was described elsewhere (7). At passage 3, the plasmid PBabe-SV40-T (neo) was transfected by electroporation $(280 \mathrm{~V}, 9601 \mathrm{~F})$ into CAFs and NPFs for cell immortalization (7). Human PCa cell lines LNCaP and nonmalignant BPH-1 cells were kind gifts from Dr. Shujie Xia of Shanghai Jiaotong University. All cells were cultured in RPMI-1640 supplemented with $10 \%$ fetal bovine serum (FBS), 100 units $/ \mathrm{ml}$ penicillin, and $100 \mu \mathrm{g} / \mathrm{ml}$ streptomycin at $37^{\circ} \mathrm{C}$ in a humidified incubator containing $5 \% \mathrm{CO}_{2}$.

Immunofluorescence assay. CAFs and NPFs $\left(10^{4}\right)$ were seeded in 4-well chamber slides and cultured overnight. The chamber slides with attached cells were routinely fixed, blocked and then incubated with the following antibodies: anti-vimentin, anti-smooth muscle aactin (SMA) (both 1:200; Sigma-Aldrich, St. Louis, MO, USA), and $\mathrm{IgG}$ control. Subsequently, slides were incubated with fluorescencelabeled secondary antibodies, and mounted in medium containing 4',6-diamidino-2-phenylindole (DAPI).

Methyl thiazolyl tetrazolium (MTT) assay. CAFs and NPFs were seeded in 24-well plates $\left(1 \times 10^{4}\right.$ cells per well $)$ and incubated with medium changed every $48 \mathrm{~h}$. The plates were stained with MTT at day 0,2, 4, 6 for $3 \mathrm{~h}$. The absorbence was read at a wave length of $575 \mathrm{~nm}$.

Malignant transformation of BPH-1 cells in vitro. The BPH-1 cells were co-cultured with CAFs or NPFs in transwell plates. CAFs or NPFs in around 50\% confluence were seeded in the lower well, and BPH-1 cells in around $30 \%$ confluence were seeded in the upper well. When the BPH-1 cells came to confluence, they were subcultured. When the CAFs and NPFs came to confluence, the cells in the lower well were exchanged with new CAFs or NPFs at around $50 \%$ confluence. The overall co-culture period was 4 weeks. The resultant BPH-1 cells were used in a soft agar assay.

Soft agar assay. Base agar ( $0.5 \mathrm{ml}$ of $1 \%$, DNA grade) was put into each well of a 6 -well plate. Then $1 \%$ agar (DNA grade agarose) was melted in a microwave prior to cooling to $40^{\circ} \mathrm{C}$ in a water bath. Agar was subsequently mixed with $3 \times$ RPMI- 1640 medium and BPH-1 cell suspension in a ratio of $1: 1: 1$, and resulted in a final cell density of $10^{5}$ cells $/ \mathrm{ml}$. Add $1 \mathrm{ml}$ of the mixture on the top of the base agar. After coagulation, add 2 ml RPMI-1640 supplemented with $10 \%$ FBS, and replace medium every 3 days to provide nutrition. After 2 weeks culture in an incubator at $37^{\circ} \mathrm{C}$, the colonies were stained by $1 \mathrm{mg} / \mathrm{ml}$ iodonitrotetrazoliumchloride solution.

Malignant transformation of BPH-1 cells in vivo. After anesthesia, five pairs of athymic male nude mice ( 8 weeks old, from Beijing Wei-tong Li-hua Laboratory Animals and Technology Ltd., Beijing, P.R. China) were put on a heating pad in the prone position. Lifting the back skin with a pair of toothed forceps, and using a coarse scissors, a $2-3 \mathrm{~cm}$ dorsal midline incision was made to expose the kidney (8). CAFs or NPFs $\left(5 \times 10^{5}\right.$ cells) and $2 \times 10^{5} \mathrm{BPH}-1$ cells were mixed in $2 \mathrm{mg} / \mathrm{ml}$ rat tail collagen (Sigma-Aldrich, St. Louis, MO, USA), then were seeded into the renal capsule of the athymic nude mice. Twelve weeks later, we sacrificed the mice and excised the kidney for further study. The care and use of nude mice in this study were approved by the Ethics Committee of the Affiliated Yantai Yuhuangding Hospital of Qingdao University (Approval No. 2013-46).
Hematoxylin and eosin $(H \& E)$ staining. Mouse xenograft tissue samples were fixed in $10 \%$ formalin and embedded in paraffin by routine. After rehydration, the sections were put into hematoxylin for 8 minutes, then rinsed in running tap water. The slides were then treated with $0.3 \%$ acid alcohol, and rinsed in running tap water followed by staining with eosin for $2 \mathrm{~min}$. The sections were dehydrated, cleared and eventually mounted.

LNCaP and CAFs/NPFs in vitro co-culture system. LNCaP cells $\left(2 \times 10^{4}\right.$ cells per well) in serum free RPMI-1640 medium supplied with $1 \mathrm{nM}$ dihydrotestosterone (DHT) were seeded in cell culture inserts of a 24-well transwell plates $(2 \mu \mathrm{m}$ microporous, from BD Biosciences, Franklin Lakes, NJ, USA). CAFs or NPFs $\left(5 \times 10^{4}\right)$ in serum-free RPMI-1640 medium were seeded in the bottom chamber. Serum-free RPMI-1640 medium was changed by $50 \%$ every $48 \mathrm{~h}$. The inserts were stained with MTT (Sigma) at day 0, 2, 4, 6 for $3 \mathrm{~h}$. The absorbance was read at a test wavelength of $575 \mathrm{~nm}$.

Invasion assay. LNCaP cells $\left(1 \times 10^{5}\right)$ in serum-free RPMI-1640 medium were added to cell culture inserts with microporous $(8 \mu \mathrm{m})$ membrane coated with or without (control insert) Matrigel (BD Biosciences, Franklin Lakes, NJ, USA). CAFs or NPFs $\left(1 \times 10^{5}\right)$ were seeded in RPMI-1640 medium containing $10 \%$ FBS into the bottom chamber. The cells were incubated for 24 hours at $37^{\circ} \mathrm{C}$, and the upper chamber was then removed. The cells on the bottom of the upper chambers were stained with $1 \%$ toluidine blue, and the number of invading cells was counted under a microscope. The invasion ratio was the ratio of the cells invading through the Matrigel-coated insert membrane to those migrating through the control non-coated insert membrane.

Xenograft model of tumor growth in vivo. Athymic male nude mice were purchased from Beijing Wei-tong Li-hua Laboratory Animals and Technology Ltd. The mixed cells were suspended in $50 \mu \mathrm{l}$ Matrigel and then implanted subcutaneously into the left flank $\left(1 \times 10^{6} \mathrm{LNCaP}\right.$ cells $\left.+1 \times 10^{6} \mathrm{CAFs}\right)$ and right flank $\left(1 \times 10^{6}\right.$ LNCaP cells $+1 \times 10^{6} \mathrm{NPFs}$ ) of nude mice to form xenograft tumors. All five mice were sacrificed at 8 weeks after inoculation. The tumors were harvested and weighed for comparison between the groups. The care and use of nude mice in this study were approved by the Ethics Committee of the Affiliated Yantai Yuhuangding Hospital of Qingdao University (Approval No. 2013-46).

Real-time quantitative polymerase chain reaction ( $Q-P C R)$. Total RNA from CAFs and NPFs was extracted and purified using Trizol (Takara, Carlsbad, CA, USA). Three micrograms of RNA was subjected to reverse transcription of genes encoding growth factors (Table I) using Superscript III (TransGene, Beijing, China). Amplification was performed using SYBR green fluorescence with the following PCR amplification conditions: 1 cycle at $95^{\circ} \mathrm{C}$ for 10 min, 45 cycles at $95^{\circ} \mathrm{C}$ for $15 \mathrm{~s}$ and $60^{\circ} \mathrm{C}$ for $60 \mathrm{~s} \mathrm{(7).} \mathrm{The} \mathrm{relative}$ expression of mRNAs was calculated using the $2^{-\Delta \Delta C T}$ method to compare the expression levels among different samples. The primer sequences are shown in Table I.

Statistical analysis. Numerical data are presented as the mean \pm standard deviation. Statistical analysis between groups was performed by using two-sided Student's $t$-test. $p$-Values of less than 0.05 were considered statistically significant. 
Table I. The primer sequences used for real-time quantitative polymerase chain reaction.

\begin{tabular}{lll}
\hline Growth factor (gene) & Sense $\left(5^{\prime}-3^{\prime}\right)$ & Antisense (5'-3') \\
\hline Insulin-like growth factor $1(I G F 1)$ & CCTCCTCGCATCTCTTCTAC & AATACATCTCCAGCCTCCTTAG \\
Placental growth factor $(P G F)$ & CTCCCCAATCCAGGCATCAA & TGGGACCCATCTTTGCTGAG \\
Epidermal growth factor $(E G F)$ & TACCGAGACCTGAAGTGG & TCTGAGTCCTGTAGTAGTGGG \\
Fibroblast growth factor $2(F G F 2)$ & GCCTTCTCTTTCAGCATTCAC & CCAACTCGTAACAATCCATCAG \\
Fibroblast growth factor $7(F G F 7)$ & CCCTGAGCGACACACAAG & CACAATTCCAACTGCCACTG \\
Fibroblast growth factor $9(F G F 9)$ & ATGGCTCCCTTAGGTGAAGTT & CCCAGGTGGTCACTTAACAAAAC \\
Fibroblast growth factor $10(F G F 10)$ & CCTCCTTCTCCTCTCCTTCC & GGCAGTTCTCCTTCTTGGTC \\
Stromal cell-derived factor $1(S D F 1)$ & CTGTGCCCTTCAGATTGTT & GGCGGAGTGTCTTTATGC \\
Hepatocyte growth factor $(H G F)$ & AGGGGCACTGTCAATACCATT & CGTGAGGATACTGAGAATCCCAA \\
Transforming growth factor $\beta 1(T G F B 1)$ & CTAATGGTGGAAACCCACAACG & TATCGCCAGGAATTGTTGCTG \\
Transforming growth factor $\beta 2(T G F B 2)$ & CCATCCCGCCCACTTTCTAC & AGCTCAATCCGTTGTTCAGGC \\
Transforming growth factor $\beta 3(T G F B 3)$ & CACCCAGGAAAACACCGAGTC & GCGGAAAACCTTGGAGGTAAT \\
Vascular endothelial growth factor B $(V E G F B)$ & GAGATGTCCCTGGAAGAACACA & GAGTGGGATGGGTGATGTCAG \\
Vascular endothelial growth factor $\mathrm{C}(V E G F C)$ & CAGTTACGGTCTGTGTCCAGTGTAG & GGACACACATGGAGGTTTAAAGAAG \\
$\beta$-Actin & CATGTACGTTGCTATCCAGGC & CTCCTTAATGTCACGCACGAT \\
\hline
\end{tabular}

\section{Results}

Primary culture and characterization of CAFs and NPFs. CAFs and NPFs were isolated from human PCa tissue and adjacent normal prostate tissue, respectively, and were immortalized with SV40-T. The cell morphologies of CAFs and NPFs were similar with elongated or stellated shapes (Figure 1A). The immunofluorescent co-staining of vimentin (fibroblast marker) and SMA (smooth muscle marker) showed the CAFs and NPFs were positive for both these markers. However, the SMA staining in CAFs was much stronger than that in NPFs (Figure 1B), which suggests the CAFs were myofibroblasts and more progenitorial than NPFs. In MTT cell growth assays, the CAFs grew faster than NPFs (Figure 1C).

BPH-1 cells were transformed into malignant cells by CAFs in vitro. For in vitro malignant transformation, BPH-1 cells were co-cultured with CAFs or NPFs in transwell plates (Figure 2A). We defined the resultant $\mathrm{BPH}-1$ cells as $\mathrm{BPH}-$ 1(CAFs) and BPH-1(NPFs). After 4 weeks co-culture, the resultant BPH-1 cells were seeded in soft agar to determine anchorage-independent colony formation ability. After 2 weeks culture, BPH-1(CAFs) were able to form colonies in soft agar, however, BPH-1(NPFs) did not form any colonies (Figure 2B).

$B P H-1$ cells were transformed into malignant cells by CAFs in vivo. BPH-1 cells combined with CAFs or NPFs in collagen were seeded into the renal capsule of nude mice. Twelve weeks later, tumors had formed in mice seeded with BPH-1 and CAFs, but not in those seeded with BPH-1 and NPFs (Figure 3A). The negative control, i.e. seeding with CAFs, NPFs, or BPH-1 cells alone did not lead to tumor formation in renal capsules of nude mice (data not shown). On H\&E staining, tumor from mice seeded with BPH-1 and CAFs presented an irregular undifferentiated malignant pattern (Figure 3B). However, the nodules from mice seeded with BPH-1 and NPFs presented a well-arranged gland-like benign pattern (Figure 3C).

CAFs promote the growth of and invasion by PCa LNCaP cells. In order to determine the role of CAFs and NPFs on PCa cell growth, LNCaP cells were co-cultured with CAFs or NPFs in transwell plates with serum-free medium supplemented with 1nM DHT (Figure 4A). In MTT assays, LNCaP cells grew extremely slowly in serum-free medium, and both CAFs and NPFs supported the growth of LNCaP cells. CAFs had a more powerful effect on growth promotion of LNCaP cells (Figure 4B). In transwell invasion assay, there were more invading LNCaP cells when co-cultured with CAFs than when co-cultured with NPFs (Figure 4C), which indicated the CAFs had a promotory effect on invasion by $\mathrm{LNCaP}$ cells.

In order to determine the role of CAFs and NPFs in vivo, we inoculated LNCaP cells combined with CAFs or NPFs subcutaneously into the flank of nude mice. Eight weeks later, the tumors from mice inoculated with LNCaP and CAFs were much bigger than those arising from inoculated with LNCaP and NPFs (Figure 4D). In summary, CAFs were more effective in promoting the growth of and invasion by $\mathrm{PCa}$ LNCaP cells both in vitro and in vivo.

CAFs secreted more growth factors than NPFs. In order to probe the mechanism of why CAFs were more effective than NPFs in promoting prostate epithelial cell transformation, growth, invasion, and tumorigenesis, we performed Q-PCR 


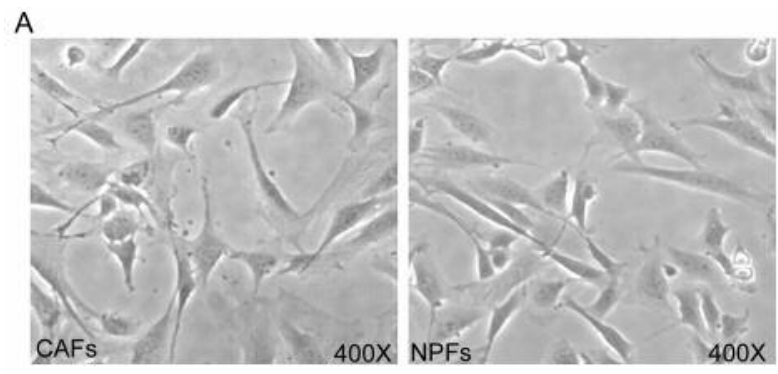

B

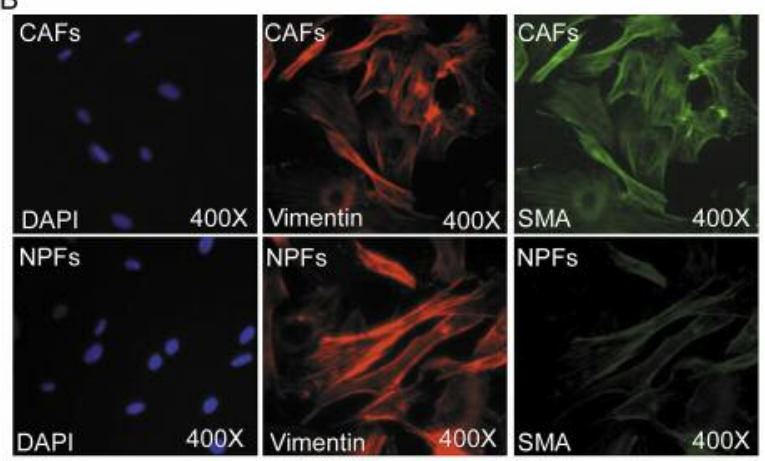

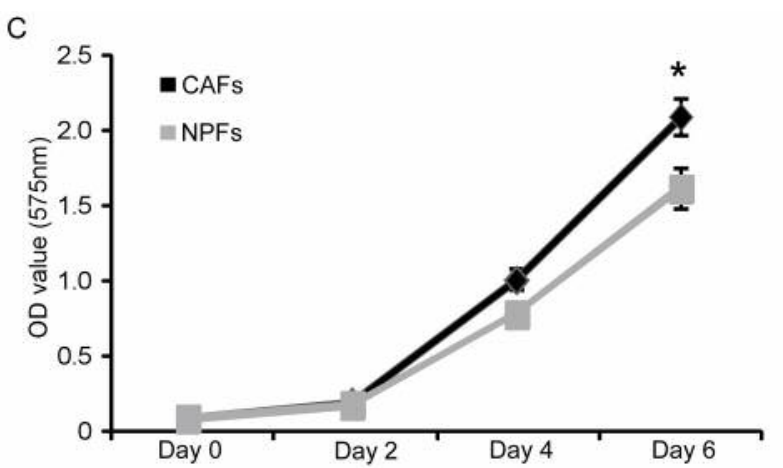

Figure 1. Primary culture and characterization of cancer-associated fibroblasts (CAFs) and normal prostate fibroblasts (NPFs). A: In phasecontrast microscopy, the morphology of CAFs and NPFs were similar, with elongated or stellated shapes. B: Immunofluorescence staining of vimentin and smooth muscle actin (SMA). SMA staining in CAFs was much stronger than that in NPFs. C: The growth curves of CAFs and $N P F s$ showed CAFs grew faster than NPFs $(n=3, * p<0.05)$.
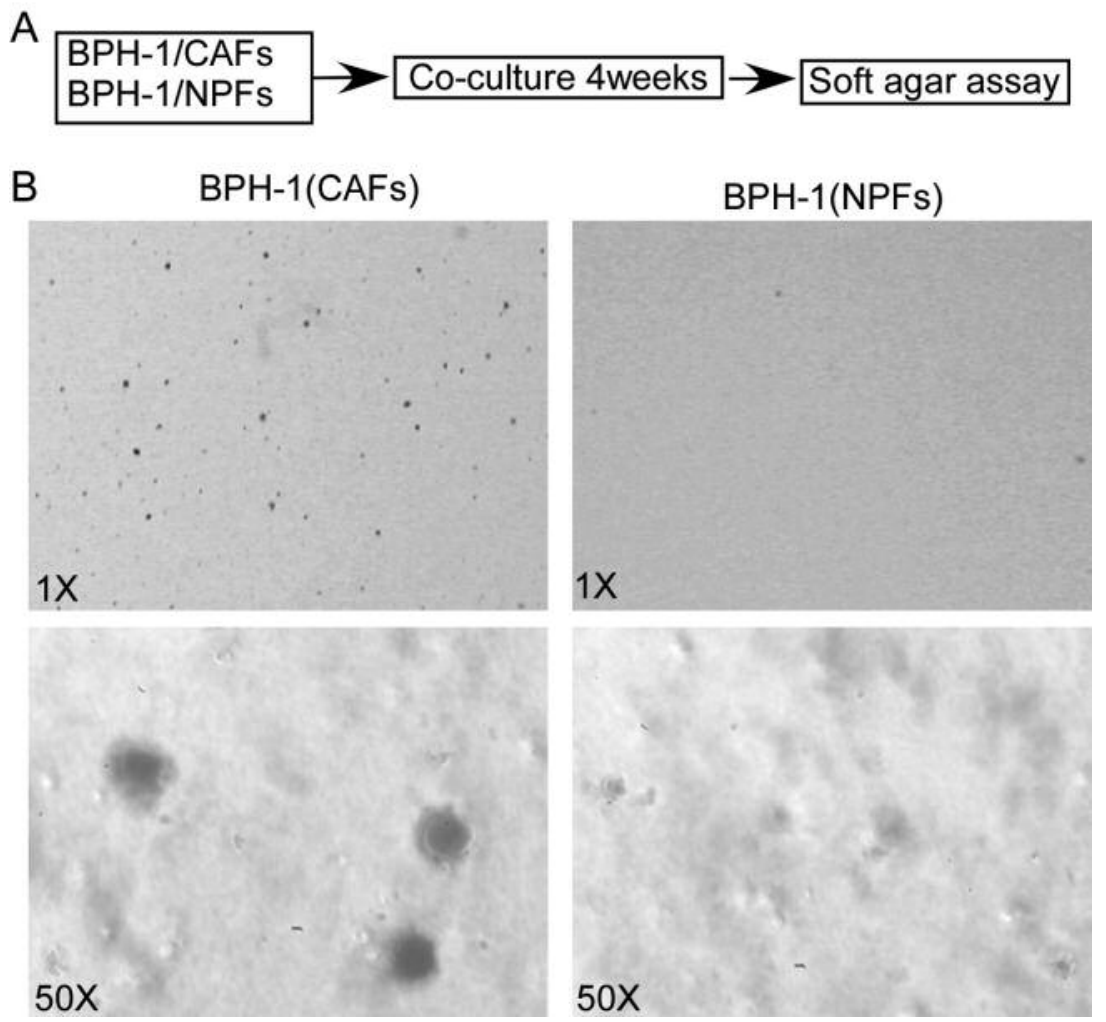

Figure 2. In vitro malignant transformation of BPH-1 cells. A: Transwell co-culture of BPH-1 cells with cancer-associated fibroblasts (CAFs) or normal prostate fibroblasts (NPFs). B: In soft agar colony formation assay, the BPH-1(CAFs) formed colonies in soft agar (left), however, the BPH$1(N P F s)$ were almost unable to form any colonies (right). 

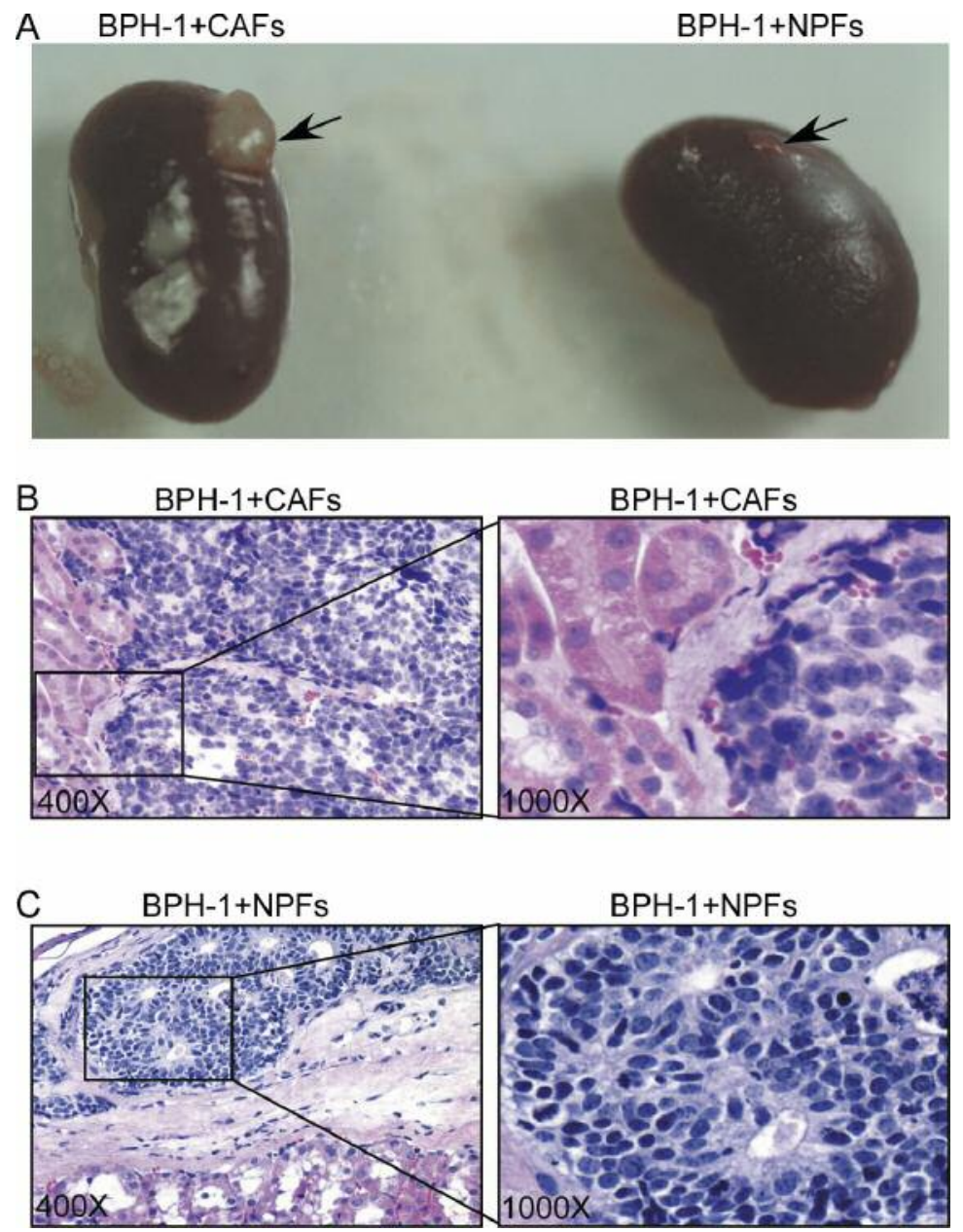

Figure 3. In vivo malignant transformation of BPH-1 cells. A: In nude mice inoculated with BPH-1 and cancer-associated fibroblasts (CAFs), tumors formed in the renal capsule (left, $n=5$ ), however, inoculation with BPH-1 and normal prostate fibroblasts (NPFs) did not lead to tumor (right, $n=5$ ). B: Hematoxylin and eosin $(H \& E)$ staining of tumor from mice inoculated with BPH-1 and CAFs presented an irregular undifferentiated malignant pattern. C: H\&E staining of a tiny nodule from mice inoculated with BPH-1 and NPFs presented a well-arranged gland-like benign pattern.

assay to screen a series of growth factors. CAFs secreted higher levels of growth factors than NPFs, namely insulin-like growth factor 1 (IGF1), placental growth factor $(P G F)$, epidermal growth factor $(E G F)$, fibroblast growth factor $(F G F)$ 2, FGF7, FGF10, stromal cell-derived factor 1 (SDF1), hepatocyte growth factor $(H G F)$, transforming growth factor $\beta 1(T G F B 1)$, transforming growth factor $\beta 3$ (TGFB3), and vascular endothelial growth factor B (VEGFB) (Figure 5).

\section{Discussion}

The cross-talk between epithelium and stroma plays critical roles in normal prostate development and cancer $(9,10)$. In tissue recombination experiments, in nude mice with a tissue recombinant of epithelia and urogenital sinus mesenchyme, prostate-like tissue formed, but did not in those with epithelia without stroma (11). During the progression of cancer, tumor cells alter the properties of the surrounding stroma to create a supportive micro-environment (12). In turn, the modified stromal cells become more active and promote the progression of cancer (10). Different types of growth factors and cytokines secreted by reactive stromal cells and the direct stroma-epithelium interactions are thought to play key roles in cancer progression (13).

PCa stroma is composed of fibroblasts, myofibroblasts, endothelial cells and immune cells (14). PCa cells produced growth factors and cytokines to activate peripheral stromal cells and resulted in the accumulation of CAFs (15). CAFs share a similar morphology with myofibroblasts observed in wound healing (13), and might originate from transformation 
A

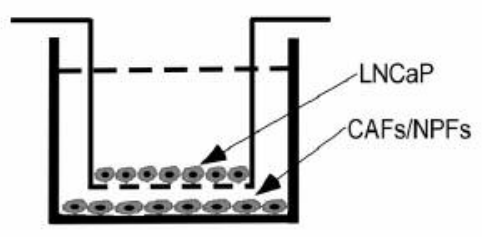

B

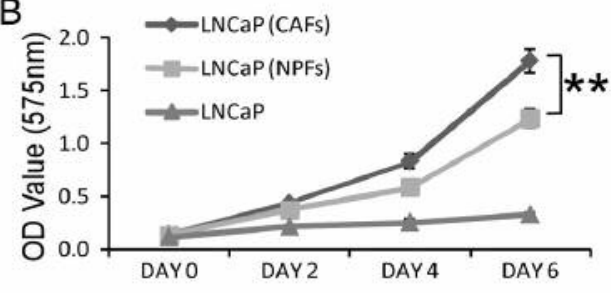

C

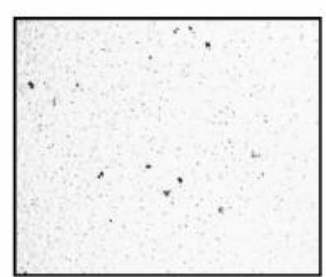

LNCaP (CAFs)

$\mathrm{D}$

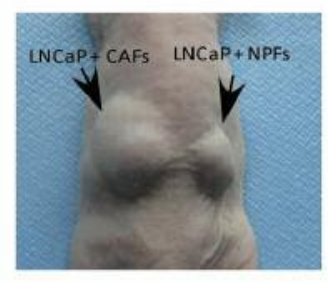

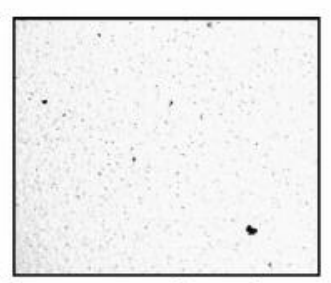

LNCaP (NPFs)

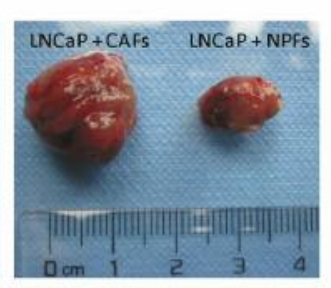

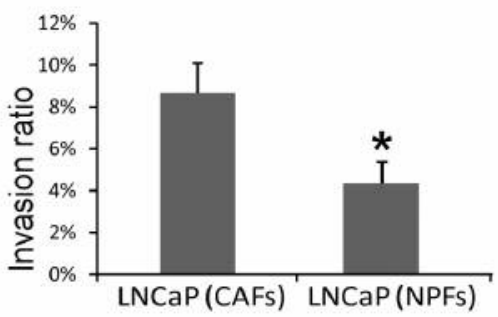

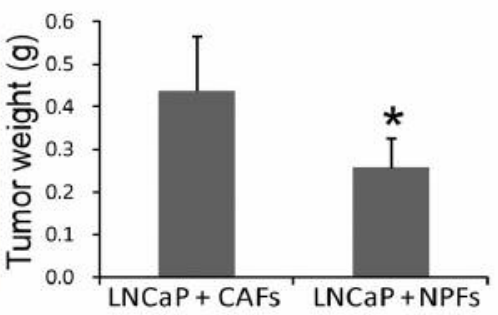

Figure 4. The effect of cancer-associated fibroblasts (CAFs) and normal prostate fibroblasts (NPFs) on the growth and invasion of LNCaP cells. A: In the co-culture transwell system, LNCaP cells were seeded in the insert of the transwell, and CAFs or NPFs were seeded on the bottom. The culture medium was serum-free RPMI-1640 supplied with $1 \mathrm{nM}$ dihydrotestosterone. B: In Methyl thiazolyl tetrazolium (MTT) assay, LNCaP cells co-cultured with CAFs grew faster than those co-cultured with NPFs $(n=3, * * p<0.01)$. C: In invasion assay, the invasion ratio was significantly higher for LNCaP (CAFs) cells than for LNCaP (NPFs) cells $(n=3, * p<0.05)$. D: LNCaP+CAFs formed larger tumors in nude mice than $L N C a P+N P F s(n=5, * p<0.05)$.

of normal fibroblasts (16), bone marrow-derived mesenchymal stem cells (17), or epithelial to mesenchymal transition (EMT) (18). CAFs are dominant components in PCa stroma $(5,6)$, and were reported to promote epithelial proliferation and invasion (19), regulate the deposition of extracellular matrix $(20,21)$, mediate inflammation and angiogenesis (22), and induce EMT and stemness of cancer cells (23).

Most previous studies used primary CAFs without immortalization. Non-immortalized CAFs can only be subcultured for 6-8 passages and may not produce enough cells for further study or for repeat experiments. Furthermore, different sets of primary cells from different PCa tissues may influence the consistency of experimental results. In the present study, we immortalized CAFs and paired NPFs from the same patient by using SV40-T, then investigated the contrasting effects of CAFs and NPFs on epithelial cells.

The morphology of immortalized CAFs and NPFs were similar with elongated or stellated shapes. The CAFs were double positive for vimentin and SMA, which was consistent with previous reports (13). However, the SMA expression level was very weak in NPFs, which means the CAFs were myofibroblasts, but the NPFs were not. The CAFs also grew faster than NPFs.

For the in vitro malignant transformation of benign prostate epithelial BPH-1 cells, we co-cultured the BPH-1 with CAFs or NPFs, producing BPH-1(CAFs) and BPH-1(NPFs) cells. The BPH-1(CAFs) cells formed colonies in soft agar, but the BPH-1 (NPFs) cells did not. Since anchor-independent growth is a property of malignant cells, the BPH-1 were transformed in vitro by CAFs, but not by NPFs. We then recombined the BPH-1 cells with CAFs and NPFs and inoculated them in the renal capsules of nude mice for in vivo transformation. Similarly, the BPH-1 with CAFs formed tumors, but the BPH1 with NPFs did not. This confirms that CAFs promote the carcinogenesis of prostate epithelial cells.

To study the effect of CAFs and NPFs on the proliferation and invasion of PCa tumors cells, we cocultured LNCaP cells with CAFs or NPFs in transwell 


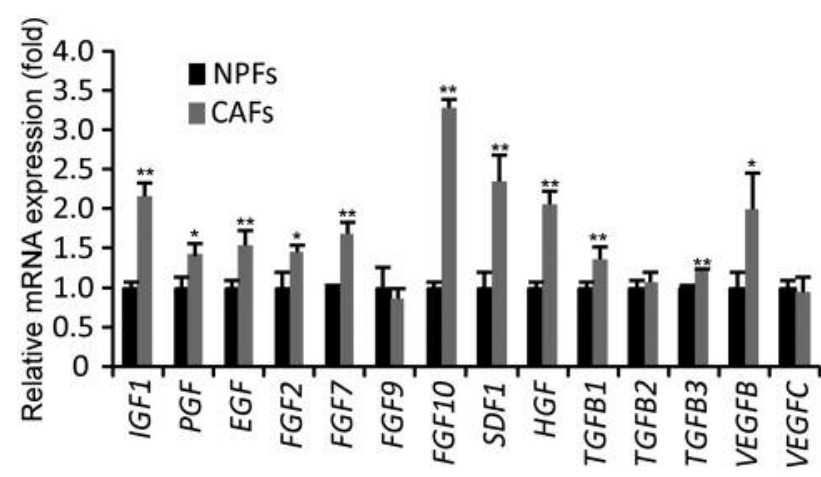

Figure 5. The mRNA expression level of multiple growth factors secreted by cancer-associated fibroblasts (CAFs) and normal prostate fibroblasts (NPFs). The expression levels of insulin-like growth factor 1 (IGF1), placental growth factor (PGF), epidermal growth factor (EGF), fibroblast growth factor (FGF) 2, FGF7, FGF10, stromal cell-derived factor 1 (SDF1), hepatocyte growth factor ( $H G F)$, transforming growth factor $\beta 1$ (TGFB1), transforming growth factor $\beta 3$ (TGFB3), and vascular endothelial growth factor $B(V E G F B)$ were higher in CAFs than those in NPFs $(n=3, * p<0.05$, ** $p<0.01)$. The expression levels of FGF9, TGFB2, and VEGFC did not significantly differ between cell types.

plates with serum free medium. The LNCaP cells cocultured with CAFs grew faster, and invaded more than the cells co-cultured with NPFs. In the subcutaneous tumor model, the tumors from LNCaP with CAFs were much bigger than those from LNCaP with NPFs. In summary, the $\mathrm{CAFs}$ were more powerful in promoting the proliferation of and invasion by $\mathrm{PCa}$ cells. To identify potential mechanism(s) we found that CAFs secreted higher levels of growth factors than NPFs. Growth factors are important for regulating a variety of cellular processes, including tumor growth, differentiation, invasion, and blood vessel differentiation.

Overall, we successfully cultured and immortalized CAFs and NPFs from the same patient, and found the CAFs secreted more growth factors, and were more active in promoting $\mathrm{PCa}$ carcinogenesis, proliferation and invasion. Targeting CAFs might be a potential option for $\mathrm{PCa}$ treatment. The immortalized CAFs and NPFs also provide good experimental tools for further PCa studies.

\section{Conflicts of Interest}

The Authors declare there are no conflicts of interest in regard to this study.

\section{Acknowledgements}

This study was supported by the National Natural Science Foundation of China (81302234), and the National Natural Science Foundation of China (81572835).

\section{References}

1 Khazaei S, Rezaeian S, Ayubi E, Gholamaliee B, Pishkuhi MA, Mansori K, Nematollahi S, Sani M and Hanis SM: Global prostate cancer incidence and mortality rates according to the Human Development Index. Asian Pac J Cancer Prev 17(8): 3793-3796, 2016.

2 Pang C, Guan Y, Li H, Chen W and Zhu G: Urologic cancer in China. Jpn J Clin Oncol 46(6): 497-501, 2016.

3 Karantanos T, Corn PG and Thompson TC: Prostate cancer progression after androgen deprivation therapy: mechanisms of castrate resistance and novel therapeutic approaches. Oncogene 32(49): 5501-5511, 2013.

4 Lee C, Jia Z, Rahmatpanah F, Zhang Q, Zi X, McClelland M and Mercola D: Role of the adjacent stroma cells in prostate cancer development and progression: synergy between TGF-beta and IGF signaling. Biomed Res Int 2014: 502093, 2014.

5 Shaw A, Gipp J and Bushman W: The Sonic Hedgehog pathway stimulates prostate tumor growth by paracrine signaling and recapitulates embryonic gene expression in tumor myofibroblasts. Oncogene 28(50): 4480-4490, 2009.

6 Schauer IG and Rowley DR: The functional role of reactive stroma in benign prostatic hyperplasia. Differentiation 82(4-5): 200-210, 2011.

7 Yu S, Xia S, Yang D, Wang K, Yeh S, Gao Z and Chang C: Androgen receptor in human prostate cancer-associated fibroblasts promotes prostate cancer epithelial cell growth and invasion. Med Oncol 30(3): 674, 2013.

8 Nicholson TM, Uchtmann KS, Valdez CD, Theberge AB, Miralem $\mathrm{T}$ and Ricke WA: Renal capsule xenografting and subcutaneous pellet implantation for the evaluation of prostate carcinogenesis and benign prostatic hyperplasia. J Vis Exp 78: 50574, 2013.

9 Cunha GR: Mesenchymal-epithelial interactions: past, present, and future. Differentiation 76(6): 578-586, 2008.

$10 \mathrm{Niu}$ YN and Xia SJ: Stroma-epithelium crosstalk in prostate cancer. Asian JAndrol 11(1): 28-35, 2009.

11 Cunha GR and Chung LW: Stromal-epithelial interactions--I. Induction of prostatic phenotype in urothelium of testicular feminized (Tfm/y) mice. J Steroid Biochem 14(12): 1317-1324, 1981.

12 Kalluri R and Zeisberg M: Fibroblasts in cancer. Nat Rev Cancer 6(5): 392-401, 2006.

13 Xing F, Saidou J and Watabe K: Cancer-associated fibroblasts (CAFs) in tumor microenvironment. Front Biosci 15: 166-179, 2010.

14 Franco OE and Hayward SW: Targeting the tumor stroma as a novel therapeutic approach for prostate cancer. Adv Pharmacol 65: 267-313, 2012.

15 Sottnik JL, Zhang J, Macoska JA and Keller ET: The PCa tumor microenvironment. Cancer Microenviron 4(3): 283-297, 2011.

16 Kojima Y, Acar A, Eaton EN, Mellody KT, Scheel C, Ben-Porath I, Onder TT, Wang ZC, Richardson AL, Weinberg RA and Orimo A: Autocrine TGF-beta and stromal cell-derived factor-1 (SDF-1) signaling drives the evolution of tumor-promoting mammary stromal myofibroblasts. Proc Natl Acad Sci USA 107(46): 20009-20014, 2010.

17 Quante M, Tu SP, Tomita H, Gonda T, Wang SS, Takashi S, Baik GH, Shibata W, Diprete B, Betz KS, Friedman R, Varro A, 
Tycko B and Wang TC: Bone marrow-derived myofibroblasts contribute to the mesenchymal stem cell niche and promote tumor growth. Cancer Cell 19(2): 257-272, 2011.

18 Thiery JP: Epithelial-mesenchymal transitions in tumour progression. Nat Rev Cancer 2(6): 442-454, 2002.

19 Kruslin B, Ulamec M and Tomas D: Prostate cancer stroma: an important factor in cancer growth and progression. Bosn J Basic Med Sci 15(2): 1-8, 2015.

20 Tomasek JJ, Gabbiani G, Hinz B, Chaponnier C and Brown RA: Myofibroblasts and mechano-regulation of connective tissue remodelling. Nat Rev Mol Cell Biol 3(5): 349-363, 2002.

21 Zhang K, Grither WR, Van Hove S, Biswas H, Ponik SM, Eliceiri KW, Keely PJ and Longmore GD: Mechanical signals regulate and activate SNAIL1 protein to control the fibrogenic response of cancer-associated fibroblasts. J Cell Sci 129(10): 1989-2002, 2016.
22 Erez N, Truitt M, Olson P, Arron ST and Hanahan D: Cancerassociated fibroblasts are activated in incipient neoplasia to orchestrate tumor-promoting inflammation in an NF-kappaBdependent manner. Cancer Cell 17(2): 135-147, 2010.

23 Giannoni E, Bianchini F, Masieri L, Serni S, Torre E, Calorini L and Chiarugi P: Reciprocal activation of prostate cancer cells and cancer-associated fibroblasts stimulates epithelial-mesenchymal transition and cancer stemness. Cancer Res 70(17): 6945-6956, 2010 .
Received May 2, 2017

Revised June 6, 2017

Accepted June 7, 2017 